\title{
Identification of High-Frequency Parameters of an Inset PM Motor Including Eddy Currents
}

\author{
Omar Bottesi, Luigi Alberti \\ Faculty of Science and Technology \\ Free University of Bozen-Bolzano \\ Bolzano (Italy) \\ omar.bottesi@natec.unibz.it
}

\author{
Ruth V. Sabariego \\ Dept. Electrical Engineering ESAT \\ Katholieke Universiteit Leuven \\ Leuven, Belgium \\ Ruth.Sabariego@esat.kuleuven.be
}

\author{
Johan Gyselinck \\ BEAMS Departement \\ Université libre de Bruxelles (ULB) \\ Bruxelles, Belgium \\ Johan.Gyselinck@ulb.ac.be
}

\begin{abstract}
This paper aims to compute the highfrequency parameters of an inset permanent magnet synchronous machine. A homogenization technique for the computation of the eddy currents and the identification of the $d-q$ model at high-frequency of the synchronous machine are briefly recalled. The simulation strategy for the Inset PM motor is introduced. A comparison between the high-frequency parameters is highlighted considering $2 \mathrm{D}$ and $3 \mathrm{D}$ models of the machine in order to validate the homogenization technique. Different operating conditions are considered: both at no load and under load. Good agreement has been found between $2 \mathrm{D}$ and $3 \mathrm{D}$ results.

Index Terms - Sensorless control, permanent magnet machines, eddy currents, finite element (FE) method, homogenization, lamination stack.
\end{abstract}

\section{INTRODUCTION}

Nowadays, advanced industrial applications require electrical drives and machines that have to be more and more reliable and compact. For this reason, sensorless applications are becoming increasingly interesting thanks to several benefits: e.g. reduction of the drive costs, increase of the fault-tolerance of the system and more compact machines. Indeed, these techniques are adopted in various applications, such as aerospace, industrial drivers, automotive, etc.

For those purposes, an evaluation of the self-sensing capability of the machine must be performed. It has been proposed for induction motors [1], [2], induction generators [3], [4], and synchronous machines [5], [6]. Therefore, there are many research works that study the self-sensing capability considering also the effect of the cross saturation. Nevertheless, there is another phenomenon which has not been totally investigated, i.e. the induction of the eddy currents in the laminations which can be further exploited to determine the rotor position with higher accuracy.

In order to assess the self-sensing capability of an electrical machine by means of signal injection, a high-frequency (hf) $d-q$ model can be adopted as in [7] and together with a finite element technique in [8], [9]. This approach consists in injecting hf sinusoidal signals superimposed at the steady state operating point. This allows to compute the $\mathrm{hf}$ inductances and resistances, even when the machine 978-1-4673-8463-6/16/\$31.00 (C) 2016 IEEE operates at zero speed. As said above, the eddy currents induced in the laminations by the hf signals may help better evaluating the self-sensing capability by including them in the FE analysis. For this purpose, there are different ways, such as the adoption of homogenization technique [10] which is employed to compute the eddy currents in the laminations of the electrical machine and which permits to reduce the computation time.

Therefore, in this paper, a $d-q$ model at high-frequency of synchronous PM machine is adopted considering homogenization technique for the computation of the eddy currents in the laminations. In particular, a comparison of the model is carried out by taking into account different polynomial orders of homogenization technique. Afterwards, the simulation procedure of an inset PM machine is introduced in order to compute the hf machine response that can be described by means of three complex parameters which form a symmetrical matrix [7]. A brief comparison between 2D and 3D models considering only a lamination thickness of the inset PM motor is presented. In addition, the hf parameters are shown for different operating conditions adopting two polynomial orders of homogenization $(n=0, n=2)$. Finally, the machine saliency and the error of the position estimation are computed [14], [15].

\section{EDDY CURRENT EQUATIONS IN A LAMINATION}

A lamination of an electrical machine is considered. The lamination is parallel to the $x y$-plane and exhibits a thickness $d$ along $z$-axis. The middle of the thickness $d$ coincides with $z=0$. The iron is assumed magnetically isotropic and nonhysteretic, and its electrical conductivity $\sigma$ is constant. The lamination carries a $\mathbf{B}$ induced by the permanent magnets as well as by the windings.

In order to compute the eddy currents induced in the lamination, the following differential equations and constitutive laws can be considered:

$$
\begin{gathered}
\nabla \times \mathbf{E}=-\frac{\partial \mathbf{B}}{\partial t}, \quad \nabla \times \mathbf{H}=\mathbf{J}, \quad \nabla \cdot \mathbf{B}=\mathbf{0}, \\
\mathbf{J}_{e d d y}=\sigma \mathbf{E}, \quad \mathbf{H}=\nu\left(\mathbf{B}^{2}\right) \mathbf{B},
\end{gathered}
$$

where $\mathbf{E}$ is the electrical field, $\mathbf{B}$ the magnetic flux density, $\mathbf{H}$ the magnetic field and $\mathbf{J}_{\text {eddy }}$ the eddy current density. 
Moreover, the reluctivity $\nu=\mu^{-1}$ is a function of $\mathbf{B}^{2}$.

In this paper $2 \mathrm{D}$ and $3 \mathrm{D}$ eddy current models are considered.

\section{A. 2D Lamination Model}

A 2D model of the electrical machine is considered. The magnetic flux density $\mathbf{B}$ and the eddy current density $\mathbf{J}_{\text {eddy }}$ in the lamination cannot explicitly computed due to the $2 \mathrm{D}$ geometry, therefore, a homogenization technique is considered [10]. The homogenization technique allows to implement 1D problems coupled with the FE equations approximating, in this case, the behaviour of the fields in the lamination. To apply that homogenization technique, the equations described in [10] have to be coupled in the formulation of the field. From the values obtained by the formulation resolution, it is possible to reconstruct the trend of $\mathbf{B}$ and $\mathbf{J}_{e d d y}$ applying the homogenization technique in the post-processing. In our case two homogenization orders have been considered: 0- and II-order and it means the magnetic flux density is considered constant and approximated with a II-order polynomial as function of $z$, respectively. 0 - and II-orders should be sufficient for approximating the magnetic field in the lamination for the considered frequencies, lamination thickness and the consequent penetration depth.

\section{B. 3D Lamination Model}

In order to validate the homogenization technique mentioned above, a 3D lamination model of the electrical machine is considered [11]. The eddy currents in the laminations are explicitly computed according to (1).

\section{Finite Element simulations of The $d-q$ HF MODEL}

The classical rotor oriented $d-q$ reference frame is considered in order to describe the machine behaviour at highfrequency. The hf voltages and currents are considered as sinusoidal small variations around the steady state operating point in order to compute the hf inductances and resistances. As assumption, the machine is operated at zero speed. Therefore, since hf injection signals is particular advantages for standstill or quasi-zero applications, the rotor is at standstill during simulations. The phasor complex notation is adopted.

The hf parameters can be defined as follows [7]:

$$
\left[\begin{array}{l}
\bar{U}_{s d} \\
\bar{U}_{s q}
\end{array}\right]=\left[\begin{array}{ll}
\dot{z}_{d d} & \dot{z}_{d q} \\
\dot{z}_{q d} & \dot{z}_{q q}
\end{array}\right]\left[\begin{array}{l}
\bar{I}_{s d} \\
\bar{I}_{s q}
\end{array}\right]
$$

where $\bar{U}_{s d}$ and $\bar{U}_{s q}$ are the hf voltages of $d$ - and $q$ axis applied to the stator terminals, respectively; $\bar{I}_{s d}$ and $\bar{I}_{s q}$ are the hf currents of $d$ - and $q$-axis at the stator terminals; while $\dot{z}_{d d}, \dot{z}_{d q}=\dot{z}_{q d}, \dot{z}_{q q}$ are the hf impedances. The hf impedances are complex numbers and they can be represented by real and imaginary parts as:

$$
\begin{array}{cc}
\dot{z}_{d d}=r_{d d}+j \omega l_{d d} & \dot{z}_{d q}=r_{d q}+j \omega l_{d q} \\
\dot{z}_{q d}=r_{q d}+j \omega l_{q d} & \dot{z}_{q q}=r_{q q}+j \omega l_{q q}
\end{array}
$$

These parameters describe the hf response of the machine at the stator terminals taking into consideration the impact of the eddy currents. They may thus be used to find out the self-sensing capability of the machine considering the effect of the parasitic phenomena such as eddy currents.

Finite element simulations have been carried out adopting the small-signal technique [8]. The machine parameters $\dot{z}_{d d}, \dot{z}_{d q}=\dot{z}_{q d}$ and $\dot{z}_{q q}$ may thus be easily computed. Such parameters are calculated in the $d-q$ frame taking into account different operating points at different frequency values as well as different angles of the rotor position.

The proposed simulation strategy is composed of:

1) bias simulation: a nonlinear magnetostatic simulation is performed in a specific working point $\left(I_{s d 0}, I_{s q 0}\right)$ and for a specific value of the rotor position $\theta_{m}$. This allows to consider the nonlinearity of the iron and all the sources in the machines, i.e. PMs and winding currents. The $\mathbf{B}_{0}$ of each element of the mesh is stored for the next step.

2) reluctivity tensor construction: from the stored $\mathbf{B}_{0}$, the differential reluctivity tensor for the smallsignal simulations is computed.

3) small-signal simulation: afterwards, a timeharmonic simulation in the frequency domain is carried out. In particular, considering different values of the injection frequency $f$ but adopting the same rotor position $\theta_{m}$ of the magnetostatic simulation. Two current driven simulations are required supplying each axis separately in order to compute the parameters $\dot{z}_{d d}, \dot{z}_{d q}=\dot{z}_{q d}$ and $\dot{z}_{q q}$.

\section{Application example}

By way of validation of the adopted homogenization technique, a particular inset PM machine is considered. This kind of machine is obtained starting from a Surface Mounted PM machine and by adding a sort of iron teeth between each couple of magnets. In Fig. 1 a sketch of the geometry of the machine is shown and, as can be noticed, the width of the iron teeth is different. This effects a reduction of the torque ripple and this technique is called shifting of the PMs [12]. In addition those iron teeth introduce anisotropy which permits a better determination of the self-sensing capability. In Table I the main data are reported. More detailed information about the machine can be found in [13].

Fig. 2 shows the magnetic flux lines in the machine at no load and at nominal load conditions. As can be noticed, the leakage flux through the iron teeth is relevant, this amounts to have a reduction of the torque because the linkage flux is lower. Nevertheless, this aspect is neglected during the analysis of the machine.

The motor has been simulated either in a $2 \mathrm{D}$ or a $3 \mathrm{D}$ model with the technique mentioned in Sec. III. In addition, in the $2 \mathrm{D}$ model the homogenization technique for the computation of the eddy currents is implemented. 


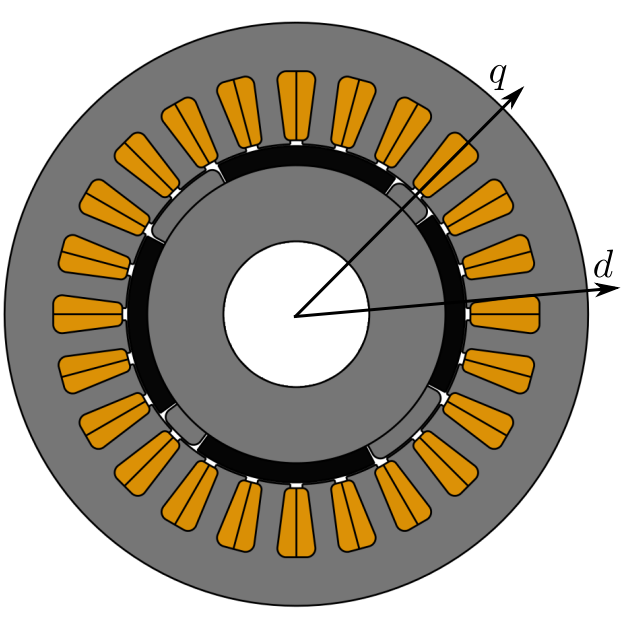

Fig. 1. Cross-section of the inset machine.

TABLE I

MAIN GEOMETRY AND MATERIAL DATA OF THE INSET MOTOR

\begin{tabular}{lll}
\hline Pole number & $2 p$ & 4 \\
Phase number & $m$ & 3 \\
Nominal Current & $I_{n}$ & $3 \mathrm{~A}$ \\
Phase resistance & $R_{p h}$ & $6.75 \Omega$ \\
Slot number & $Q_{s}$ & 24 \\
Fill factor & $k_{f i l l}$ & 0.5 \\
Air gap & $g$ & $0.3 \mathrm{~mm}$ \\
External diameter & $D_{e}$ & $120 \mathrm{~mm}$ \\
Inner diameter & $D_{i}$ & $70 \mathrm{~mm}$ \\
Slot height & $h_{s}$ & $15 \mathrm{~mm}$ \\
Back iron height & $h_{b i}$ & $10 \mathrm{~mm}$ \\
Stack length & $L_{s t k}$ & $40 \mathrm{~mm}$ \\
lamination thickness & $d$ & $0.65 \mathrm{~mm}$ \\
PM coercitive field & $H_{c}$ & $-629 \mathrm{kA} / \mathrm{m}$ \\
\hline
\end{tabular}

\section{A. hf parameters comparison of $2 D$ and $3 D$ models}

At first, the $2 \mathrm{D}$ model is validated comparing the $\mathrm{hf}$ parameters with the values computed through 3D simulations. Several linear simulations with frozen reluctivity have been carried out considering an injection frequency range form $100 \mathrm{~Hz}$ to $2 \mathrm{kHz}$. The rotor is at standstill and the $d$-axis of the rotor is aligned with the $d$-axis of the windings. The motor works at no load, the currents in the magnetostatic simulations are thus zero. The eddy currents in the PMs are neglected. In the 3D model, only one lamination of thickness $d$, obtained by extruding the 2D geometry along the thickness $d$, is analyzed. In Fig. 3 a detail of the lamination fo the $3 \mathrm{D}$ model is shown. The surface and thickness discretisation is highlighted.

Fig. 4 shows the hf inductive parameters versus the frequency. As can be noticed, the trend of the hf parameters is expected due to the variation of the penetration depth $\delta$ with the frequency. Therefore, the higher frequency, the lower magnetic flux, and consequently inductances. The trend of the 0-order results is totally different due to the low order of approximation. A good agreement between the 3D and II-order results is shown. Nevertheless, a slight

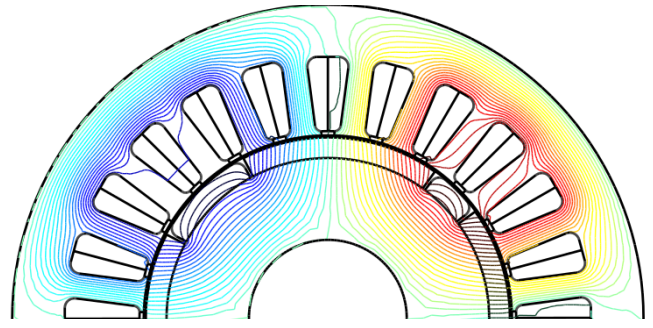

(a) no load.

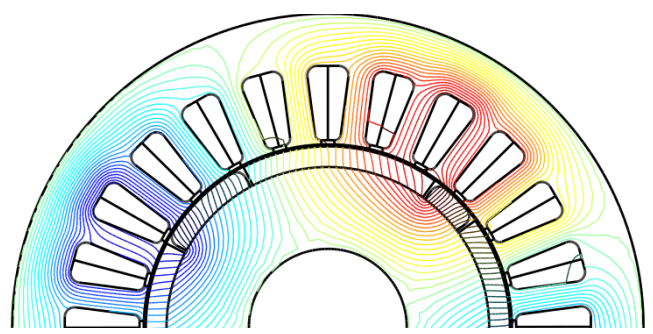

(b) nominal load.

Fig. 2. Magnetic flux lines in the inset PM motor.

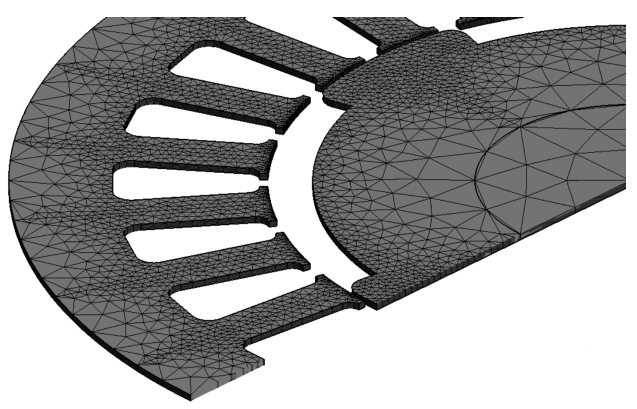

Fig. 3. Detail of the lamination in the 3D model of the inset machine.

difference is present due to the $3 \mathrm{D}$ effects. $l_{q q}$ is higher than $l_{d d}$ due to the iron teeth between the PMs which are centered on the $d$-axis.

Fig. 5 depicts the $d$ - and $q$-axis hf resistive parameters versus the frequency. The resistive parameters increases with the frequency due to the higher losses in the laminations. As for the inductive parameters, the 3D and II-order results are in good agreement, while the 0 -order results are quite different. $r_{q q}$ is higher than $r_{d d}$ for the same reason as for the inductive parameters.

\section{B. 2D Finite Element Simulations considering different operating conditions}

After the assessment of the homogenization technique with the comparison with the $3 \mathrm{D}$ model, several 2D nonlinear simulations have been performed taking into account different operating conditions. The eddy currents in the PMs are taken into account and they are explicitly computed. The injection of the hf currents occurs by way of coupled circuits with the FE model. It is thus easy to compute the $d$ - and $q$-axis voltages which are necessary for calculating the hf parameters. The $2 \mathrm{D}$ simulations are 


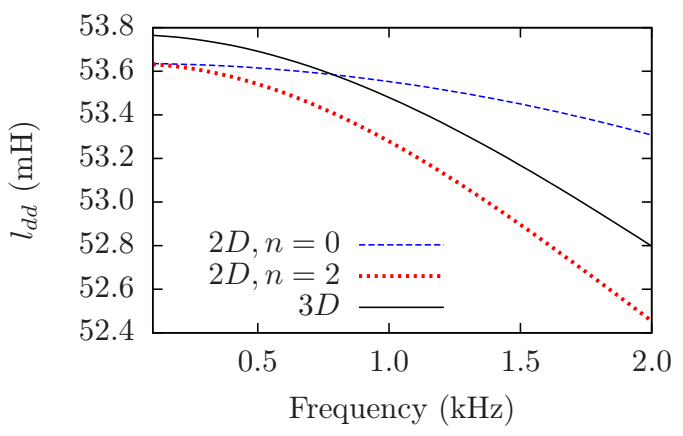

(a) $d$-axis inductive parameter.

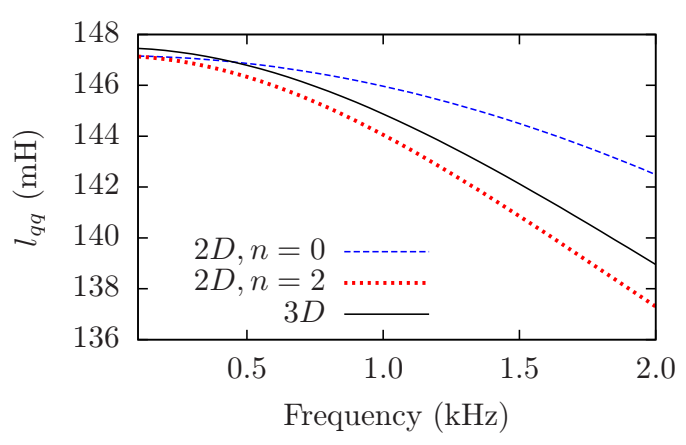

(b) $q$-axis inductive parameter.

Fig. 4. hf inductive parameters vs the injection frequency at no load. Comparison between 2D (considering 0- and II-order homogenization) and 3D models

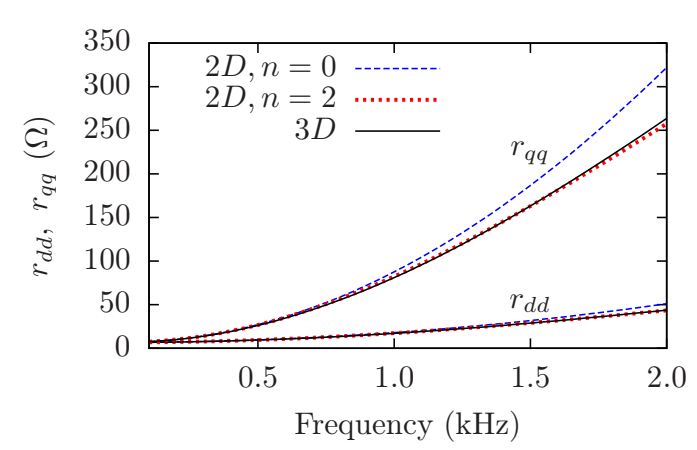

Fig. 5. $d$ - and $q$-axis hf resistive parameters vs the injection frequency at no load. Comparison between $2 \mathrm{D}$ and $3 \mathrm{D}$ models considering $0-$ and II-order homogenization

performed as follows:

- the machine is operated in two different conditions: no load and under load, with $I_{s d 0}=0 \mathrm{~A}, I_{s q 0}=0 \mathrm{~A}$ and $I_{s d 0}=0 \mathrm{~A}, I_{s q 0}=3 \mathrm{~A}$, respectively.

- the injection frequency is set for each operating condition. The considered values are $100 \mathrm{~Hz}, 200 \mathrm{~Hz}$, $400 \mathrm{~Hz}, 600 \mathrm{~Hz}, 800 \mathrm{~Hz}, 1000 \mathrm{~Hz}$.

- for each operating condition and frequency, the angle of the rotor position is varied between 0 and 60 degree in order to assess the periodicity of the machine which is of 30 mechanical degree.
- consequently, for each rotor position, the current of $d$ - and $q$-axis are driven separately.

- finally, the procedure is repeated for the 0- and IIorder homogenization technique.

Fig. 6 represents the hf inductive parameters versus the rotor position for no load and under load operations. The results are achieved considering a $f=400 \mathrm{~Hz}$. Looking at Fig. 6(a), a slight difference between the results obtained with $n=0$ and $n=2$ can be seen when the machine is in no load. Furthermore, the values of $l_{d d}$ and $l_{q q}$ decrease due to the saturation. Fig. 6(b) shows the $l_{d q}=l_{q d}$. It is worth noticing that $l_{d q}=l_{q d}$ and they decrease with the saturation assuming negative values due to the sign of the mutual coupling.

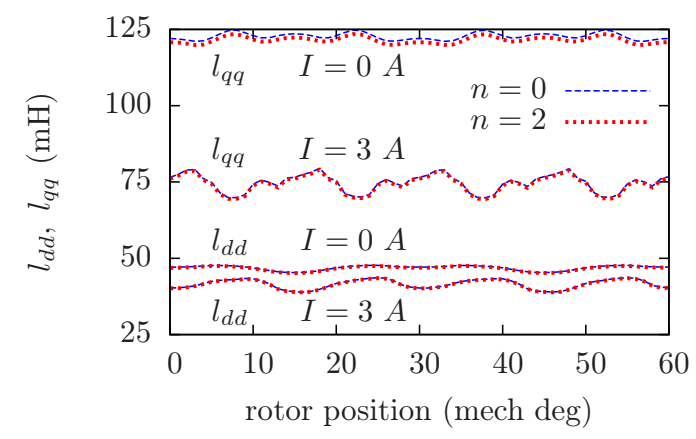

(a) $d$ and $q$-axis inductive parameters.

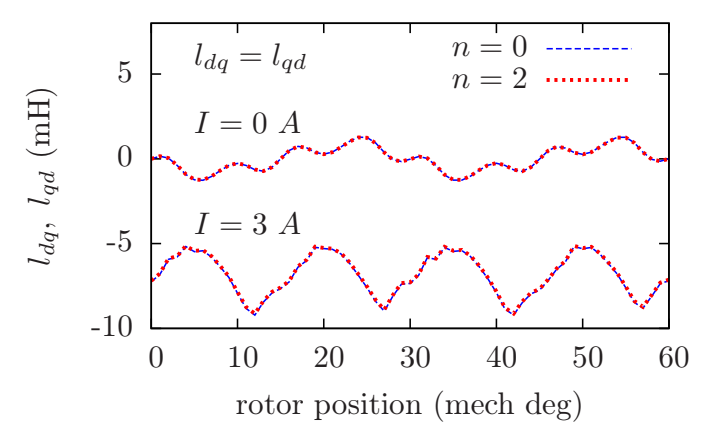

(b) cross-coupling inductive parameters.

Fig. 6. hf inductive parameters vs the rotor position for no load and under load operations and considering two orders of homogenization approximation. Injection frequency of $400 \mathrm{~Hz}$

Fig. 7 shows the hf resistive parameters vs the rotor position when the injection frequencies are 400 and $1000 \mathrm{~Hz}$ in no load condition. Fig. 7(a) exhibits $r_{d d}$ which increases with frequency as expected. The difference between the $n=0$ and $n=2$ increases with frequency in agreement to Fig. 5. The same behaviour can be seen in Fig. 7(b) for $r_{q q}$ whose value is much higher due to the iron teeth between the PMs.

A more interesting case is reported in Fig. 8 which shows the cross-coupling hf resistive parameters versus the rotor position for 400 and $1000 \mathrm{~Hz}$ injection frequencies under load condition. The $r_{d q}=r_{q d}$ are negative due to the sign 


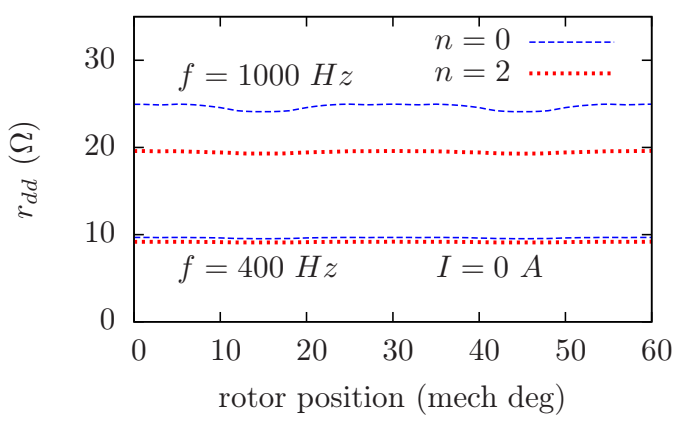

(a) $d$-axis resistive parameters.

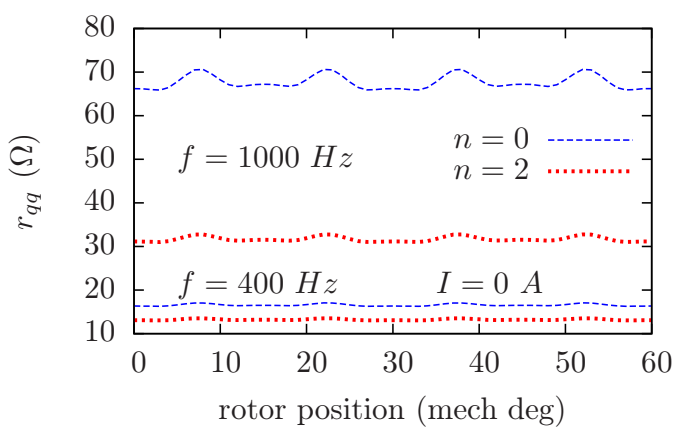

(b) $q$-axis resistive parameters.

Fig. 7. hf resistive parameters vs the rotor position for 400 and $1000 \mathrm{~Hz}$ injection frequencies in no load condition.

of the mutual coupling and they become higher and more oscillating with the load due to the saturation. In this case a relatively high difference can be seen between the 0 - and II-order homogenization.

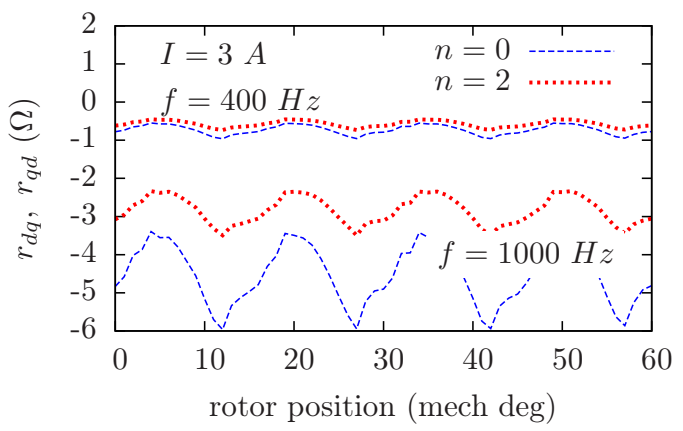

Fig. 8. cross-coupling hf resistive parameters vs the rotor position for 400 and $1000 \mathrm{~Hz}$ injection frequencies in under load condition.

Finally, Fig. 9 shows the $q$-axis hf resistive parameters vs the rotor position for $400 \mathrm{~Hz}$ injection frequency in no load and under load conditions. In comparison with Fig. 6(a) a strong effect of the 0 - and II-order homogenization is exhibited in the resistive parameters due to the eddy currents induced in the laminations. This effect can be also seen in Fig. 5. The same behaviour occurs in the $d$ axis resistive parameter $r_{d d}$.

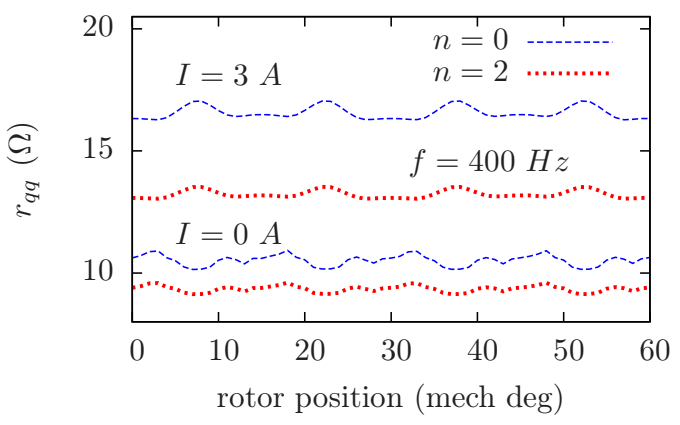

Fig. 9. $q$-axis hf resistive parameters vs the rotor position for $400 \mathrm{~Hz}$ injection frequency in no load and under load conditions.

To sum up, the hf resistive parameters are strongly affected by the injection frequency because the losses due to the eddy currents depend on the $f^{2}$, whereas the $\mathrm{hf}$ inductive parameters are not strongly influenced by the injection frequency. Therefore, the eddy currents little affect the hf inductive parameters. In addition, the 0order homogenization at high injection frequencies is not sufficient due to the considerable skin effect.

\section{Computation of the machine saliency and the error in the position estimation}

When $d$ - and $q$-axis currents are not orthogonal due to the mutual coupling between the $d$ - and $q$-axis, symmetrical components can be adopted to investigate the selfsensing capability of the machine [14], [15]. Therefore, both $d$ - and $q$-axis currents can be written as $\bar{i}_{s d}=\bar{i}_{s d}^{f}+\bar{i}_{s d}^{b}$ and $\bar{i}_{s q}=\bar{i}_{s q}^{f}+\bar{i}_{s q}^{b}$ where $\bar{i}_{s d}^{f}$ and $\bar{i}_{s q}^{f}$ are the forward components and $\bar{i}_{s d}^{b}$ and $\bar{i}_{s q}^{b}$ the backward components. The machine saliency $\xi$ can be then computed as:

$$
\xi=\frac{\left|\bar{i}_{s d}^{f}\right|+\left|\bar{i}_{s d}^{b}\right|}{\left|\bar{i}_{s d}^{f}\right|-\left|\bar{i}_{s d}^{b}\right|}
$$

The angle error $\varepsilon$ can be obtained through:

$$
\varepsilon=\frac{\arg \left(\bar{i}_{s d}^{f}\right)-\arg \left(\bar{i}_{s d}^{b}\right)}{2}
$$

Fig. 10 shows the machine saliency and the error in the position estimation versus the rotor position in no load and under load condition at $400 \mathrm{~Hz}$ for 0- and IIorder homogenization. As can be seen from Fig. 10(a), the saliency $\xi$ is well above one and it ensures a good tracking of the rotor position. As expected, there is a saliency oscillation with the rotor position which is considerable under load due to the saturation. In Fig. 10(b) the error angle $\varepsilon$ is shown. At no load condition the error oscillates around zero, while at nominal load it increases.

There is no apparent difference in the 0- and II-order results due to the resistive part of the hf parameters which can be neglected with respect the inductive part. It 
permits to understand that the eddy currents mainly affect the hf resistive parameters. If the machine were resistivebased salient, the difference would be visible.

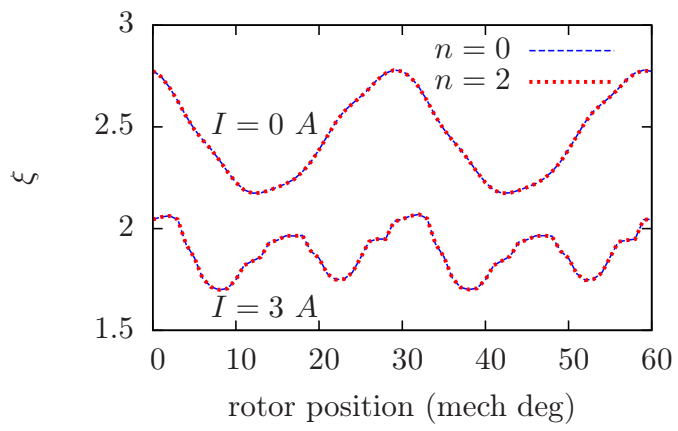

(a) Machine saliency $\xi$.

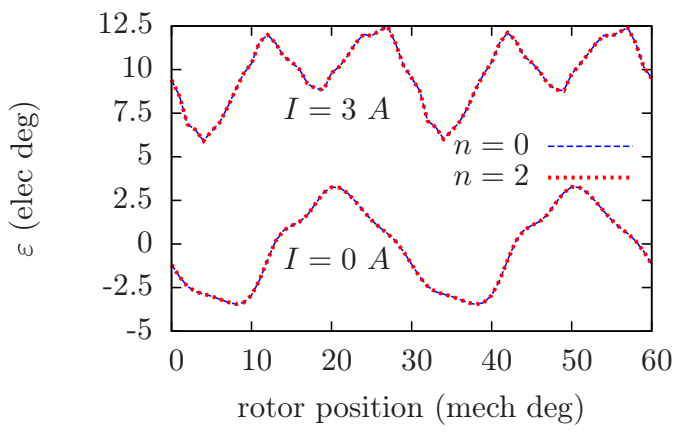

(b) Position error $\varepsilon$.

Fig. 10. Machine saliency and position error vs the rotor position in no load and under load condition considering an injection frequency of $400 \mathrm{~Hz}$

\section{Conclusions}

In this paper, the computation of the hf parameters of an inset PM machine is presented, in particular, taking into account the eddy currents in the laminations and in the PMs. Moreover, the eddy currents in the laminations are implemented considering a 0 - and II-order homogenization technique.

A comparison between the 2D and 3D models of the inset PM machine is presented and there is a good agreement between the II-order homogenization 2D and 3D results. It is shown that the adoption of a $2 \mathrm{D}$ model with proposed homogenization technique allows to reduce the computational time and the model complexity. Furthermore, the hf parameters for different operating condition and rotor position are presented highlighting the effect of the saturation. If the degree of saturation increases, the 0 -order homogenization does not manage to approximate correctly.

Finally, the machine saliency and the error of the position estimation are computed. The machine presents a saliency higher then 1 . Such a condition is important for a good tracking of the rotor position. In the considered machine, the hf resistive parameters can be neglected with respect the inductive one.

\section{REFERENCES}

[1] Degner, M. W. and Lorenz, R. D., "Using multiple saliencies for the estimation of flux, position, and velocity in AC machines", IEEE Transactions on Industry Applications, vol. 34, no. 5, pp.1097-1104, Sep./Oct. 1998.

[2] Holtz, J., "Sensorless position control of induction motors-an emerging technology", IEEE Transactions on Industrial Electronics, vol. 45, no. 6, pp. 840-851, Dec. 1998.

[3] Iwanski, G. and Koczara, W., "Sensorless Direct Voltage Control of the Stand-alone Slip-ring Induction Generator", IEEE Transactions on Industrial Electronics, vol. 54, no. 2, pp. 1237-1239, Apr. 2007.

[4] Pena, R., Cerdenas, R., Proboste, J., Asher, G., and Clare, J., "Sensorless Control of Doubly-fed Induction Generators Using a Rotor-current-based MRAS Observer", IEEE Transactions on Industrial Electronics, vol. 55, no. 1, pp. 330-339, Jan. 2008.

[5] Consoli, A., Russo, F., Scarcella, G., and Testa, A., "Low-and zero-speed sensorless control of synchronous reluctance motors", IEEE Transactions on Industry Applications, vol. 35, no. 5, pp. $1050-1057$, sep/oct 1999.

[6] Jang, J.-H., Sul, S.-K., Ha, J.-I., Ide, K., and Sawamura, M., "Sensorless drive of surface-mounted permanent-magnet motor by high-frequency signal injection based on magnetic saliency", IEEE Transactions on Industry Applications, vol. 39, no. 4, pp.1031 - 1039, july-aug. 2003.

[7] Alberti, L.; Bianchi, N. \& Bolognani, S. "High Frequency d-q Model of Synchronous Machines for Sensorless Control", IEEE Transactions on Industry Applications, 2015, PP, 1-1

[8] Alberti, L.; Gyselinck, J.; Bianchi, N.; Morandin, M. \& Bolognani, S. "Small-signal finite-element modeling of synchronous machines for sensorless applications", 2012 XXth International Conference on Electrical Machines (ICEM), 2012, 2266-2272

[9] Alberti, L.; Bianchi, N.; Morandin, M.; Gyselinck, J.J.C., "Finite-Element Analysis of Electrical Machines for Sensorless Drives With High-Frequency Signal Injection," in IEEE Transactions on Industry Applications, vol.50, no.3, pp.1871-1879, May-June 2014

[10] Gyselinck, J.; Sabariego, R. \& Dular, P. "A nonlinear timedomain homogenization technique for laminated iron cores in three-dimensional finite-element models" in IEEE Transactions on Magnetics, 2006, 42, 763-766

[11] Gyselinck, J.; Geuzaine, C. V. S. R. "Considering Laminated Cores and Eddy Currents in 2D and 3D Finite Element Simulation of Electrical Machines" in 18th Conference on the Computation of Electromagnetic Fields (COMPUMAG2011), 2011,

[12] Bianchi, N.; Bolognani, S., "Design techniques for reducing the cogging torque in surface-mounted PM motors," in IEEE Transactions on Industry Applications, vol.38, no.5, pp.12591265, Sep/Oct 2002

[13] Bianchi, N., Bolognani, S., Jang, J.-H., and Su,l S.-K., "Advantages of inset pm machines for zero-speed sensorless position detection", IEEE Transactions on Industry Applications, vol. 44, no. 4, pp. $1190-1198$, july-aug. 2008.

[14] Alberti, L.; Bianchi, N.; Bolognani, S., "Investigation on the self-sensing capability of a fractional-slot inset PM motor", 2013 IEEE International Symposium on Sensorless Control for Electrical Drives and Predictive Control of Electrical Drives and Power Electronics (SLED/PRECEDE), 2013, pp. 1-6

[15] Albert,i L.; Bianchi, N.; Bolognani, S. "Comparison of different synchronous machines for sensorless drives", IECON 2013 - 39th Annual Conference of the IEEE Industrial Electronics Society, 2013, 8220-8226

[16] Yang, S.-C. and Lorenz, R. D., "Comparison of resistance-based and inductance-based self-sensing control for surface permanent magnet machine using high frequency signal injection", in Energy Conversion Congress and Exposition (ECCE), 2011 IEEE, sept. 2011, pp. $2701-2708$.

[17] Peter, H. and Hahn, I., "Determination of differential inductances of permanent magnet synchronous machines for sensorless control", in IEEE International Electric Machines Drives Conference (IEMDC), May 2011, pp. 1579-1584. 